\title{
Inclusão de ácidos graxos de cadeia curta em diferentes níveis de cálcio na dieta de frangos de corte
}

\author{
Inclusion of short chain fatty acids and different calcium levels in diets for broilers
}

\author{
Maitê de Moraes Vieira ${ }^{\mathrm{I}}$ Alexandre de Mello Kessler ${ }^{\mathrm{II}}$ Andréa Machado Leal Ribeiro ${ }^{\mathrm{II}}$
}

\section{RESUMO}

Noventa e seis frangos de corte, machos, de linhagem Cobb e de 21 aos 31 dias de idade foram alimentados com a inclusão de ácidos graxos de cadeia curta (AGCC) $e$ diferentes níveis de cálcio. Foram determinados o balanço de cálcio (Ca) e de fósforo (P), a metabolizabilidade da matéria seca (MetMS), matéria orgânica (MetMO) e matéria mineral, da energia bruta e proteína bruta da dieta, a porcentagem de cinzas nas tíbias (\%CzT), além do desempenho das aves. Utilizouse um esquema fatorial $5 \times 4$ (sem ácido orgânico; ácido fórmico, ácido acético, ácido propiônico e ácido butírico) x (0,40, 0,59, 0,78 e $0,97 \%$ de Ca), com um delineamento completamente casualizado. O acréscimo de Ca na dieta afetou de forma positiva e quadrática o balanço de Ca e P, sendo os melhores níves estimados em 0,87 e $0,75 \%$ de Ca dietético, respectivamente, e afetou positiva e linearmente a MetMS e a MetMO, bem como a \%CzT. Houve redução na eficiência de retenção do Ca, de forma quadrática, com o aumento do nível de cálcio. Os AGCC não apresentam efeitos detectáveis sobre as respostas estudadas e nem interação com os níveis dietéticos de Ca utilizados. A melhor exigência estimada de Ca dietético, considerando o balanço de cálcio, foi de $0,87 \%$.

Palavras-chave: ácidos orgânicos, exigência de cálcio, mineralização óssea.

\section{ABSTRACT}

Ninety six Cobb male broilers from 21 to 31 days of age were fed including short chain fatty acids (SCFA) and different level of calcium. Calcium (Ca), phosphorus (P), balance, dry, organic and mineral matter metabolizability, crude energy and crude protein metabolizability, tibia ash percentage and animal performance were measured. Experimental diets were assigned in a 5x4 factorial arrangement (without acids, formic acid, acetic acid, propionic acid and butyric acid) $x$
(0.40, 0.59, 0.78 and $0.97 \%$ of $\mathrm{Ca}$ ) in a randomized completely design. Increasing Ca levels affected positively and in a quadratic form Ca and $P$ balance and the best levels were estimated at $0,87 \%$ and $0,75 \%$ of Ca dietary, respectively and affected linearly dry, organic matter metabolizability and tibia ash percentage. However, the efficiency of Ca retention was decreased quadratically with the increase of Ca level. The SCFA did not show detectable effects on the studied responses, and no interaction with dietary levels of $\mathrm{Ca}$ used. The estimated Ca requirement considering calcium balance was $0.87 \%$.

Key words: organic acids, calcium requirement, bone mineralization.

\section{INTRODUÇÃO}

Os ácidos orgânicos são utilizados na formulação de dietas para aves com o objetivo de interferir no trato gastrintestinal, modificando a microbiota e/ou acidificando o meio luminal e trazendo efeitos benéficos sobre a digestibilidade dos nutrientes (inclusive minerais) ou o controle de microrganismos indesejáveis.

O estudo da acidificação da dieta para aves tem um histórico recente. Alguns autores têm tentado demonstrar o efeito dos ácidos graxos de cadeia curta no desempenho de frangos de corte (VALE et al., 2004; LEESON et al., 2005; HERNANDEZ et al., 2006). Porém, a ação benéfica dos ácidos graxos de cadeia curta no metabolismo digestivo das aves ainda não foi completamente elucidada.

IPrograma de Pós-graduação em Zootecnia, Universidade Federal do Rio Grande do Sul (UFRGS), Porto Alegre, RS, Brasil.

IIDepartamento de Zootecnia, UFRGS. Av. Bento Gonçalves, 7712, 91540-000, Porto Alegre, RS, Brasil. E-mail: aribeiro@ufrgs.br.

*Autor para correspondência. 
A influência no transporte intestinal de cálcio foi verificada in vitro por MINEO et al. (2001), que, utilizando ácido acético, propiônico e butírico em preparações de tecido da mucosa do cólon de ratos, comprovaram o efeito positivo dos ácidos graxos de cadeia curta no mecanismo de transporte paracelular de cálcio no intestino.

Diversos autores têm pesquisado sobre as exigências de cálcio nas dietas para frangos de corte (SÁ et al., 2004; DRIVER et al., 2005; ROSTAGNO et al., 2005). Entretanto, as estimativas dessas exigências são um pouco divergentes em função do critério considerado para estipular o nível de cálcio na dieta, buscando a melhor resposta dos animais, mas em média valores em torno de 1\% têm sido estabelecidos, o que está muito próximo do estabelecido pelo NRC (1994). A necessidade de otimizar a suplementação do cálcio nas dietas para frangos de corte estimula a busca por um nível ótimo, pois a inclusão excessiva desse mineral ocupa um valioso espaço nas formulações de ração e pode até, em alguns casos, prejudicar o desempenho dos animais (SCHOULTEN et al., 2003).

Um gradiente de concentração de cálcio mínimo entre a mucosa e a serosa intestinal é necessário para a absorção eficiente de cálcio solúvel no ceco e no cólon proximal e distal (RASCHKA \& DANIEL, 2005). A presença de grande quantidade de ácidos graxos de cadeia curta no ceco, principalmente acetato, butirato e propionato, proporciona a diminuição do pH intraluminal (CAMPBELL et al., 1997) e o aumento da solubilidade do cálcio (LOPEZ et al., 1998). Como resultado, há um aumento no gradiente de concentração, promovendo a entrada passiva de cálcio através do transporte transepitelial (RASCHKA \& DANIEL, 2005). Entretanto, apesar das evidências do aumento do transporte de cálcio no intestino grosso, pela ação dos ácidos de origem fermentativa, nesse local ocorre a menor parte da absorção do cálcio da dieta (BRONNER \& PANSU, 1999), tornando pequena essa contribuição. Consequentemente, deve-se investigar se efeito semelhante ocorre no intestino delgado. Com a otimização da utilização do cálcio da dieta, possibilita-se menor inclusão desse mineral na formulação desta.

O objetivo deste trabalho foi avaliar o efeito de diferentes ácidos graxos de cadeia curta (AGCC), em dietas com diferentes níveis de cálcio, sobre a retenção aparente de cálcio e fósforo e o metabolismo ósseo de frangos de corte.

\section{MATERIAL E MÉTODOS}

Foram utilizados 96 frangos de corte Cobb, dos 21 aos 31 dias de idade, mantidos em uma sala climatizada, com temperatura mínima média de $20^{\circ} \mathrm{C}$ e máxima de $24^{\circ} \mathrm{C}$ e um programa de luz de 24 horas. Os animais foram alojados em gaiolas individuais com comedouro individual, bebedouro tipo calha e bandeja para a coleta individual de excretas. A água e ração foram fornecidas à vontade, com controle individual de consumo de ração.

As dietas foram formuladas com níveis nutricionais próximos daqueles recomendados pelas Tabelas Brasileiras para Aves e Suínos (ROSTAGNO et al., 2005), sendo isoproteicas (20\%PB) e isoenergéticas (3100 $\mathrm{kcal} \mathrm{kg}^{-1}$ ), diferindo somente no nível de cálcio e na adição dos diferentes AGCC. Para a produção das dietas experimentais, foi utilizada uma única dieta basal, sendo os diferentes níveis de cálcio obtidos pela inclusão de calcário calcítico - 37\% cálcio (substituído por areia fina), além da inclusão dos diferentes AGCC, que foram usados na forma pura e substituídos por amido de milho. Foi avaliada a inclusão equimolar (20meq $\mathrm{kg}^{-1}$ ) (MINEO et al., 2001) dos ácidos fórmico (0,09\%), acético (0,12\%), propiônico $(0,15 \%)$ e butírico $(0,18 \%)$ em dietas com níveis crescentes de cálcio $(0,40 ; 0,59 ; 0,78$ e $0,97 \%)$, constituindo um fatorial $5 \times 4$ e totalizando 20 tratamentos em um delineamento completamente casualizado (tratamentos com $0,97 \% \mathrm{Ca}$, com quatro repetições, e os demais NC com cinco repetições cada).

Os animais foram adaptados às instalações durante quatro dias, quando todos receberam a mesma dieta. As respostas de desempenho avaliadas durante o experimento foram ganho de peso, consumo de ração e conversão alimentar. O controle do consumo de ração e a coleta total de excretas foram realizados dos 21 aos 31 dias de idade para determinação da metabolizabilidade da matéria seca (MetMS), da matéria orgânica (MetMO), da matéria mineral (MetMM), da proteína bruta (MetPB) e da energia bruta (MEB) da dieta e do balanço aparente de cálcio (BCa) e fósforo (BP) (determinados pela diferença dos nutrientes consumidos e excretados). Os balanços aparentes de Ca e $P$ foram avaliados nas formas absoluta (em gramas) e relativa (em porcentagem do ingerido). A primeira representa o total de nutriente absorvido ao longo do período, e a segunda, a eficiência de retenção do nutriente. Aos 31 dias de idade, todos os animais foram abatidos para coleta da tíbia esquerda e avaliação da porcentagem de cinzas na tíbia, na matéria seca (\%CzT), como indicador de mineralização óssea. 
As análises de matéria seca, cinzas e nitrogênio das rações e excretas, bem como de cinzas nas tíbias foram realizadas de acordo com a AOAC (1995). Para a análise das cinzas, as tíbias foram secas a $105^{\circ} \mathrm{C}$, por 12 horas, e queimadas em mufla a $550^{\circ} \mathrm{C}$, por quatro horas, sem prévia extração da gordura. A energia bruta das dietas e das excretas seguiu os procedimentos de calorimetria usando uma bomba calorimétrica da PARR INSTRUMENTS. Co. (1988). As análises de Ca e $P$ nas rações e excretas foram realizadas por espectrometria de absorção atômica e colorimetria, conforme especificações de TEDESCO et al. (1995).

O modelo estatístico foi analisado por análise de variância pelo método dos Quadrados Mínimos Generalizados, com o programa computacional Statgraphics plus 4.1 (MANUGISTICS, 1997). As médias dos fatores principais foram comparadas pelo teste de Student-Newman-Keuls, a 5\% de probabilidade. O peso inicial dos animais foi incluído como covariável nas análises. O efeito dos níveis de cálcio foi testado de forma contínua por análise de regressão. As estimativas de exigência de cálcio foram calculadas a partir das equações de regressão das respostas estudadas (segunda derivada no caso de equações quadráticas e nível máximo testado no caso de respostas lineares).

\section{RESULTADOS E DISCUSSÃO}

Os níveis de cálcio (NC) utilizados mostraram um efeito significativo na metabolizabilidade da matéria seca e da matéria orgânica das dietas (Tabela 1). Na MetMS, a regressão foi linear e positiva (MetMS $=63,69+4,57 * N C ; \mathrm{R}^{2}=0,16 ; \mathrm{P}<0,001$ ), indicando melhora no aproveitamento de nutrientes à medida que aumentou o NC. Uma explicação para esse efeito pode estar relacionada à formulação das dietas experimentais, já que o aumento dos NCa veio acompanhado da redução de areia fina. O Ca da dieta vai para os ossos, não sendo recuperado nas fezes, diferentemente do diluente usado. Por outro lado, a melhora concomitante e linear do aproveitamento da matéria orgânica (MetMO=66,73+4,84*NC; $\mathrm{R}^{2}=0,16 ; \mathrm{P}<0,001$ ) pode ser um indicativo que o aumento de cálcio tenha favorecido a digestão de componentes dietéticos outros que não os minerais, provavelmente pela via fermentativa, em função de possíveis alterações no $\mathrm{pH}$ intestinal (VIEIRA et al., 2008). Entretanto, a forma pela qual o cálcio afeta a melhora na metabolizabilidade da dieta ainda precisa ser esclarecida.

Não foram verificadas diferenças significativas entre os tratamentos estudados na metabolizabilidade da matéria mineral, indicando que não houve diferença quanto à proporção do total de minerais absorvidos em cada tratamento. No entanto,

Tabela 1 - Efeito dos níveis de cálcio (NC) e da adição de ácidos graxos de cadeia curta (AGCC) sobre a metabolizabilidade da matéria seca (MetMS), da matéria orgânica (MetMO), da matéria mineral (MetMM), da proteína bruta (MetPB) e da energia bruta (MetEB) das dietas em frangos de corte machos de 21 a 31dias de idade.

\begin{tabular}{|c|c|c|c|c|c|}
\hline Fator & MetMS(\%) & MetMO(\%) & MetMM(\%) & MetPB(\%) & MetEB(\%) \\
\hline \multicolumn{6}{|c|}{ 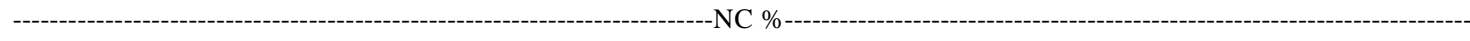 } \\
\hline 0,40 & 65,51 a & 68,55 a & 23,33 & 58,63 & 72,05 \\
\hline 0,59 & $66,72 \mathrm{ab}$ & $69,74 \mathrm{ab}$ & 25,25 & 58,82 & 72,93 \\
\hline 0,78 & $67,38 \mathrm{~b}$ & $70,52 \mathrm{~b}$ & 24,80 & 58,67 & 73,21 \\
\hline 0,97 & $68,15 \mathrm{~b}$ & 71,33 b & 24,74 & 60,20 & 73,65 \\
\hline Fórmico & 67,02 & 70,09 & 24,76 & 59,45 & 73,06 \\
\hline Acético & 66,35 & 69,46 & 23,49 & 57,71 & 72,73 \\
\hline Propiônico & 66,72 & 69,82 & 24,39 & 58,30 & 72,79 \\
\hline Butírico & 67,15 & 70,17 & 25,82 & 59,58 & 72,90 \\
\hline Sem ácidos & 67,47 & 70,62 & 24,22 & 60,36 & 73,33 \\
\hline NC & 0,004 & 0,002 & 0,519 & 0,415 & 0,126 \\
\hline AGCC & 0,680 & 0,664 & 0,658 & 0,158 & 0,935 \\
\hline NCxAGCC & 0,587 & 0,567 & 0,715 & 0,262 & 0,444 \\
\hline CV (\%) & 3,70 & 3,48 & 19,36 & 6,05 & 3,20 \\
\hline
\end{tabular}

*Médias na mesma coluna seguidas de letras distintas diferem entre si (Teste SNK, $\mathrm{P}<0,05$ ). 
essa resposta não indica diretamente se houve alguma interferência na quantidade de cálcio absorvida pelos animais, visto que, no metabolismo dos minerais, uma fração é absorvida no intestino, enquanto outra é perdida via urina, estando presente nas excretas das aves. Nesse sentido, é possível que a excreção conjunta de fezes e urina, característica nas aves, tenha prejudicado a detecção de diferenças entre tratamentos, em função da falta de mensuração das perdas urinárias.

Não foram detectadas diferenças significativas na metabolizabilidade da proteína bruta e energia bruta da dieta, tanto em relação ao cálcio, quanto à inclusão de AGCC (Tabela 1). Da mesma forma, SEBASTIAN et al. (1996) não verificaram efeito do nível de Ca da ração na retenção de nitrogênio em frangos de corte de um a 21 dias de idade. Ao contrário, SCHOULTEN et al. (2003) observaram que baixos níveis de cálcio $(0,46 \%)$ reduziram a retenção de nitrogênio em frangos dessa mesma idade.

Os AGCC não afetaram a mineralização óssea dos frangos, pois não houve diferenças significativas na porcentagem de cinzas na tíbia (Tabela 2). No entanto, diferentemente do resultado do presente estudo, SNOW et al. (2004) e RAFACZ-LIVINGSTON et al. (2005) verificaram o aumento nas cinza da tíbia de frangos de corte alimentados com dietas contendo ácido cítrico. Por outro lado, o NC da dieta interferiu na deposição mineral óssea das aves, havendo efeito linear dos NC sobre a porcentagem de cinza da tíbia na matéria seca (\%CzT=31,03+8,18*NC; $\left.\mathrm{R}^{2}=0,35 ; \mathrm{P}<0,001\right)$. Esses resultados estão de acordo com o trabalho de SÁ et al. (2004), onde os valores de cinza dos ossos em porcentagem elevaram-se com o aumento de cálcio na dieta. Entretanto, discordam dos resultados de ALVES et al. (2002) e SCHOULTEN et al. (2003) onde o aumento do NC não afetou a deposição mineral na tíbia de aves em fase de crescimento.

A inclusão de AGCC também não interferiu de forma significativa no balanço de Ca e P (Tabela 2). Da mesma forma, VALENCIA \& CHAVEZ (2002), em um experimento com suínos, não observaram efeito na retenção de cálcio quando o ácido acético foi adicionado na dieta. Porém, MROZ et al. (2000) observaram, em suínos, que a retenção de cálcio foi afetada pelos ácidos orgânicos fórmico, butírico e fumárico, e a retenção de fósforo interagiu com a capacidade tamponante da dieta. Em frangos de corte, na fase inicial, a utilização de dietas contendo ácido citrico melhorou significativamente a retenção de cálcio e fósforo (RAFACZ-LIVINGSTON et al., 2005; BRENES et al., 2003).

Já os diferentes NC influenciaram o balanço de Ca e P, sendo a absorção absoluta (gramas) desses minerais afetada de forma quadrática e positiva - BCa $\mathrm{g}=-3,62+22,76 * \mathrm{NC}-13,03 * \mathrm{NC}^{2}\left(\mathrm{R}^{2}=0,49 ; \mathrm{P}<0,001\right) ; \mathrm{BP}$ $\mathrm{g}=2,77+5,92 * \mathrm{NC}-3,97 * \mathrm{NC}^{2}\left(\mathrm{R}^{2}=0,12 ; \mathrm{P}<0,001\right)$. A eficiência na retenção do fósforo (BP \%) melhorou de forma linear com o aumento dos níveis dietéticos de

Tabela 2 - Efeito dos níveis de cálcio (NC) e da adição de ácidos graxos de cadeia curta (AGCC) sobre o balanço do cálcio (BCa) e do fósforo (BP) dietéticos e a porcentagem de cinzas na tíbia expressos na matéria seca (\%CzT) e a respectiva exigência estimada de cálcio para frangos de corte machos de 21 a 31 dias de idade.

\begin{tabular}{|c|c|c|c|c|c|}
\hline Fator & $\mathrm{BCa}(\mathrm{g})$ & $\mathrm{BCa}(\%)$ & $\mathrm{BP}(\mathrm{g})$ & BP (\%) & $\% \mathrm{CzT}$ \\
\hline \multicolumn{6}{|c|}{ - } \\
\hline 0,40 & $3,69 a^{*}$ & 57,00 a & $4,54 \mathrm{a}$ & 46,65 a & 34,15 a \\
\hline 0,59 & $5,34 \mathrm{~b}$ & 54,95 b & 4,79 a & 49,18 b & $36,61 \mathrm{~b}$ \\
\hline 0,78 & $6,09 \mathrm{c}$ & $49,31 \mathrm{c}$ & 4,89 a & $49,81 \mathrm{~b}$ & 37,92 bc \\
\hline 0,97 & $6,50 \mathrm{c}$ & $41,60 \mathrm{c}$ & 4,95 a & $50,05 \mathrm{~b}$ & 38,73 c \\
\hline Fórmico & 5,38 & 51,95 & 4,66 & 48,77 & 37,12 \\
\hline Acético & 5,55 & 50,41 & 4,97 & 49,09 & 37,38 \\
\hline Propiônico & 5,48 & 51,37 & 4,85 & 49,21 & 36,37 \\
\hline Butírico & 5,42 & 50,62 & 4,82 & 49,17 & 36,87 \\
\hline Sem ácidos & 5,19 & 49,23 & 4,67 & 48,00 & 36,52 \\
\hline NC & 0,001 & 0,001 & 0,055 & 0,013 & 0,001 \\
\hline AGCC & 0,674 & 0,746 & 0,374 & 0,894 & 0,702 \\
\hline NCxAGCC & 0,999 & 0,991 & 0,551 & 0,139 & 0,679 \\
\hline CV (\%) & 14,54 & 12,89 & 11,50 & 8,74 & 6,65 \\
\hline Exig. Ca (\%) & 0,87 & 0,46 & 0,75 & $=0,97$ & $=0,97$ \\
\hline
\end{tabular}

*Médias na mesma coluna seguidas de letras distintas diferem entre si (Teste SNK, $\mathrm{P}<0,05)$. 
cálcio $\left(\mathrm{BP} \%=44,79+5,70 * \mathrm{NC} ; \mathrm{R}^{2}=0,08 ; \mathrm{P}<0,001\right)$, diferentemente do trabalho de ALVES et al. (2002). Considerando que nível de fósforo dietético no presente experimento foi constante entre os tratamentos (0,38\% Pdisp), esse resultado parece estar relacionado à uma menor excreção urinária de fósforo nas dietas com alto cálcio.

A eficiência na retenção de cálcio (BCa\%) diminuiu de forma quadrática à medida que aumentaram os níveis de cálcio da dieta $(\mathrm{Bca} \%=44,65+53,21 * \mathrm{NC}-$ $\left.57,69 * \mathrm{NC}^{2} ; \mathrm{R}^{2}=0,69 ; \mathrm{P}<0,001\right)$. A regulação intestinal da absorção de cálcio é influenciada pela quantidade desse mineral presente na dieta. Quando o consumo de cálcio é alto, sua absorção é baixa e vice-versa (GANONG, 2003). Com baixos níveis de cálcio na dieta, a absorção intestinal de Ca através do transporte transcelular torna-se primordial, responsabilizando-se por uma substancial fração do que é absorvido no intestino delgado. Nesse caso, o canal de membrana (TRVP6) e a proteína ligadora de cálcio (calbindin-D), ambos limitantes, tornam-se reguladores (BRONNER, 2003). Com consumos altos, o Ca passa a ser transportado principalmente pela via paracelular ou transporte passivo e, nesse caso, depende da solubilidade do mesmo, da permeabilidade do epitélio intestinal e do tempo de passagem luminal (BROONER, 1998). A menor eficiência de retenção de cálcio com o aumento dos níveis dietéticos pode ser devido à regulação, através do menor nível de Ca, da síntese da proteína ligadora de $\mathrm{Ca}$ na mucosa intestinal (HURWITZ et al., 1995). Além disso, pode ter ocorrido uma redução da capacidade absortiva em função de um maior tamponamento do ambiente luminal pelos maiores níveis de carbonato de cálcio no intestino ou ainda essa redução no aproveitamento de Ca ser devido à maior excreção urinária desse mineral. Por outro lado, ARAÚJO et al. (2002) não observaram modificações na excreção de cálcio com sua diminuição na dieta de 0,90 para $0,68 \%$.

A estimativa de exigência de cálcio calculada a partir das análises de regressão das respostas estudadas contempla valores próximos das recomendações publicadas, visto que estas oscilam entre 0,75 (BP g) e 1,01\% (\%CzT). A exceção é a estimativa de $0,46 \%$ de Ca dietético para $\mathrm{BCa}(\%)$, e isso indica que a quantidade necessária para otimizar o aproveitamento de cálcio está bem abaixo do nível necessário para a manutenção da calcemia do organismo, em função da ineficiência do processo metabólico. Já a estimativa de exigência de cálcio para balanço de cálcio (em gramas) foi de $0,87 \%$ de cálcio dietético, sendo essa a resposta que apresentou o melhor coeficiente de determinação.
Embora de importância secundária para os objetivos do presente experimento, o desempenho médio dos animais, no período total, foi de $1550 \pm 12 \mathrm{~g}$ de consumo de ração, $879 \pm 9 \mathrm{~g}$ de ganho de peso e $1,77 \pm 0,01$ em conversão alimentar. Não foram verificadas diferenças significativas nessas respostas para os níveis de Ca ou a adição de AGCC na dieta $(\mathrm{P}>0,05)$, o que vai ao encontro dos resultados obtidos por SÁ et al. (2004), que usaram 0,41 a 1,41\% de Ca na dieta. Da mesma forma, ARAÚJO et al. (2002) verificaram que a redução do nível de cálcio na dieta, de 0,9 para $0,68 \%$, para frangos de corte na fase de crescimento, não afetou o desempenho. A ausência de efeito da adição dos AGCC na dieta concorda com diversos autores que utilizaram esses ácidos graxos nessa espécie. GARCIA et al. (2000), com 0,2\% de uma mistura de ácido fórmico propiônico, LEESON et al. (2005), com ácido butírico (0,2 e 0,4\%), e recentemente HERNANDEZ et al. (2006) e GARCÍA et al. (2007), com ácido fórmico (0,5 e 1\%) na dieta, não encontraram diferenças no desempenho.

\section{CONCLUSÃO}

Ácidos graxos de cadeia curta, adicionados à dieta de frangos de corte, não afetaram a exigência de cálcio, a mineralização óssea e a metabolizabilidade dos nutrientes. O aumento do nível de cálcio na dieta melhorou o aproveitamento de cálcio e fósforo, sendo 0,87\% o melhor nível para a fase de crescimento.

\section{REFERÊNCIAS}

ALVES, E.L. et al. Efeito dos níveis de cálcio sobre o desempenho de frangos de corte. Revista Ciência e Agrotecnologia, v.26, n.6, p.1305-1312, 2002. Disponível em: <http:// www.editora.ufla.br/revista/26_6/art25.PDF>. Acesso em: 2 set. 2006.

ARAÚJO, C.S.S. et al. Desempenho, rendimento de carcaça e excreção de cálcio de frangos de corte alimentados com diferentes níveis de aminoácidos e cálcio no período de 22 a 42 dias de idade. Revista Brasileira de Zootecnia, v.31, n.6, p.2209-2215, 2002. Disponível em: <http://www.scielo.br/ s c i elo.ph p ? s c ri pt = s ci__art text \& pid = S 1516 $35982002000900008 \& \operatorname{lng}=\mathrm{en} \& \mathrm{nrm}=\mathrm{iso} \& \mathrm{t} \operatorname{lng}=\mathrm{pt}>$. Acesso em: 2 out. 2006. doi: 10.1590/S1516-35982002000900008.

ASSOCIATION OF OFFICIAL AGRICULTURAL CHEMISTIS (AOAC). Official Methods of Analysis. 16 ed. Washington, 1995. 1141p.

BRENES, A. et al. The effect of citric acid and microbial phytase on mineral utilization in broiler chicks. Animal Feed Science and Technology, v.110, p.201-219. 2003. Disponível em: <http:/ /www.sciencedirect.com/science?_ob=ArticleURL\&_udi=B6T4249J8WSW-1\&_user $=687304 \&$ _coverDate $=11 \% 2 \mathrm{~F} 11 \% 2 \mathrm{~F} 200$ 3\&_alid $=1008611821 \&$ \&doc $=1 \&$ \&mt $=$ high\&_orig $=$ search 
\&_cdi $=4962 \&$ \&sort $=r \&$ \& docanchor $=\&$ view $=$ c $\&$ _ct $=1$ \&_acct $=$ C $000037798 \&$ \&_version $=1 \&$ \&_urlVersion $=0 \&$ _userid=687304\&md5=5173e90da2c7e2ec43b55fbf356e380c $>$. Acesso em: 26 nov. 2008. doi:10.1016/S0377-8401(03)00207-4.

BRONNER, F. Mechanisms of intestinal calcium absorption. Journal Cell Biochemical, v.88, n.2, p.387-393, 2003. Disponível em: <http://www3.interscience.wiley.com/cgi-bin/ fulltext/101520388/HTMLSTART>. Acesso em 11 nov. 2006. doi: $10.1002 /$ jcb.10330.

BRONNER, F.; PANSU, D. Nutritional aspects of calcium absorption. Journal of Nutrition, v.129, p.9-12, 1999. Disponível em: <http://jn.nutrition.org/cgi/content/full/129/1/ 9 ? $\mathrm{m}$ a $\mathrm{xt}$ o s h o w $=\& \mathrm{H} \mathrm{I} \mathrm{T} \mathrm{S}=10$ \& h i t s $=10$ \& R E S U L T F O R M A T $=\&$ s e a r c h i d $=1$ \& F I R S T I N D E X $=0$ \& s o r t s p e c $=$ rele vance \& volu me $=129 \&$ firstpage $=9 \&$ resourcetype $=$ HWCIT $>$. Acesso em: 27 fev. 2007.

BRONNER, F. Calcium absorption - a paradigm for mineral absorption. Journal of Nutrition, v.128, p.917-930, 1998. Disponível em: <http://jn.nutrition.org/cgi/content/full/128/5/ 917 ? $\mathrm{maxt}$ os how $=\&$ H I T S $=10 \&$ hit s $=10 \&$ R E S ULT FORMAT $=\&$ andorexactfulltext=and \&searchid= $1 \&$ F I R S T I N D E X $=0$ \& s o r t s p e c $=$ re le vance $\&$ volume $=128 \&$ firstpage $=917 \&$ resourcetype $=$ HWCIT $>$. Acesso em: 11 nov. 2006.

CAMPBELL, J.M. et al. Selected indigestible oligosaccharides affect large bowel mass, cecal and fecal short-chain fatty acids, $\mathrm{pH}$ and microflora in rats. Journal of Nutrition, v.127, p.130136, 1997. Disponível em: <http://jn.nutrition.org/cgi/content/ full $/ 127 / 1 / 130$ ? maxtoshow $=\&$ HITS $=10$ \&hits $=10 \&$ RESUL TFORMAT $=\&$ andorexactfulltext $=$ and $\&$ searchid $=$ $1 \&$ F I R S T I N D E X $=0$ \& s or t s p e c $=$ rele v a n c e \& volume $=127 \&$ firstpage $=130 \&$ resourcetype $=$ HWCIT $>$. Acesso em 11 nov. 2006.

DRIVER, J.P. et al. Calcium requirements of modern broiler chicken as influenced by dietary protein and age. Poultry Science, v.84, p.1629-1639, 2005. Disponível em: <http://ps.fass.org/cgi/reprint/ $84 / 10 / 1629$ ? $\mathrm{maxt}$ os how $=\&$ H I T S $=10 \&$ hits $=10 \&$ R E S U L T F O R M A T $=\&$ s e a r c h i d $=1$ \& F I R S T I N D E X $=0$ \& s o r t s p e c $=$ re le va n c e \& v o l u me $=84$ \& firstpage $=1629$ \&resourcetype $=$ HWCIT $>$. Acesso em: 29 set. 2006

GARCÍA, F. et al. Effect of formic acid and plant extracts on growth, nutrient digestibility, intestine mucosa morphology, and meat yield of broilers. Journal of Applied Poultry Research, v.16, p.555-562, 2007. Disponível em: <http://japr.fass.org/cgi/ content/full/16/4/555? maxtoshow=\&HITS=10\&hits=10\&RES U L T F O R M A T $=\&$ s e a r c h i d $=1 \&$ F I R S T I N D E X $=0 \&$ s ort s p e c $=$ r e l e va n c e \& vol ume $=16 \&$ firstpage $=555 \&$ resourcetype $=$ HWCIT $>$. Acesso em: 2 jul. 2007. doi:10.3382/japr.2006-00116.

GARCIA, R.G. et al. Isolated or combinated action of organic acids and growth promoter in broilers rations. Revista Brasileira de Ciência Avícola, v.2, n.2, p.149-154, 2000. Disponível em: $<$ http://www.scielo.br/scielo.php?script=sci_arttext\&pid=S1516635X2000000200004\&lng=en\&nrm=iso>. Acesso em: $27 \mathrm{fev}$. 2007. doi: 10.1590/S1516-635X2000000200004.

GANONG, W.F. Review of medical physiology. 31.ed. San Francisco: Lange Medical Book, 2003. 912p.
HERNANDEZ, F. et al. Effect of formic acid on performance, digestibility, intestinal histomorphology and plasma metabolite levels of broiler chickens. British Poultry Science, v.47, n.1, p.50-56, 2006. Disponível em: <http://web.ebscohost.com/ ehost/pdf?vid=7\&hid=8\&sid=cd3fe 8cf-699b-4c2d-a1525ae2833eb902\%40sessionmgr14>. Acesso em: 9 mar. 2008. doi: $10.1080 / 00071660500475574$.

HURWITZ, S. et al. Calcium metabolism and requirements of chickens are affected by growth. Journal of Nutrition, v.125, p.2679-2686, 1995. Disponível em: <http://jn.nutrition.org/cgi/ reprint $/ 125 / 10 / 2679$ ? maxtoshow $=\&$ HITS $=10 \&$ hits $=10 \&$ RES ULTFORMAT=\&andorexactfulltext=and\&searchid= $1 \&$ F I R S T I N D E X $=0$ \& s or t s p e c $=$ re le v a n c e \& volume $=125 \&$ firstpage $=2679 \&$ resourcetype $=H W C I T>$. Acesso em: 3 ago. 2007.

LEESON, S. et al. Effect of butiric acid on the performance and carcass yield of broiler chickens. Poultry Science, v.84, p.1418-1422, 2005. Disponível em: <http://ps.fass.org/cgi/ reprint $/ 84 / 9 / 1418$ ? maxtoshow $=$ \&HITS $=10$ \&hits $=10 \&$ RESU L T F O R M A T = \& a n d o rex a c t f u l l t e x t $=$ a n d \& s e a r c h i d $=1 \&$ F I R S T I N D E X $=0$ \& s orts p e c $=$ relevance $\&$ volume $=84 \&$ firstpage=1418\&resourcetype=HWCIT $>$. Acesso em: 24 jan. 2006.

LOPEZ, H.W. et al. Intestinal fermentation lessens the inhibitory effect of phytic acid on mineral utilization in rats. Journal of Nutrition, v.128, p.1192-1198, 1998. Disponível em: <http:/ / j n . nutrition.org/cgi/content/full/ $128 / 7$ / 1192 ? $\mathrm{maxt}$ oshow $=\&$ HITS $=10 \&$ hits $=10 \& \mathrm{RESULT}$ FORMAT $=\&$ andorexactfulltext $=$ and $\&$ searchid $=1 \&$ F I R S T I N D E X $=0$ \& s or t s p e c $=$ re l e va n c e \& vo lume $=128 \&$ firstpage $=1192 \&$ resourcetype $=$ HWCIT $>$. Acesso em: 3 ago. 2007.

MANUGISTICS. Statgraphics plus for Windows. (versão 4.1). Rockville, Maryland, 1997. CD-ROM.

MINEO, H. et al. Short-chain fatty acids enhance diffusional $\mathrm{Ca}$ transport in the epithelium of the cecum and colon. Life Science, v.69, p.517-526, 2001. Disponível e m : < h t t p : / / w w w. s c i e n c ed i re c t. c o m/ science?_ob=ArticleURL\&_udi=B6T99-43CB8SC$3 \&$ \&user $=687304 \&$ _coverDate $=06 \% 2 F 22 \% 2 F 2001 \&$ alid $=101$ 2579180\&_rdoc $=1 \&$ \& fmt $=$ high\&_orig $=$ search\&_cdi $=5109 \&$ _ sort=r\&_docanchor $=\&$ view $=$ c\&_ct=1\&_acct $=$ C000037798\&_version $=1 \&$ \& u r l Versi o n $=0 \&$ _ u s e r i d $=68730$ 4\&md5=0c54902a84eeb1ea361207bc60894e91>. Acesso em: 30 jun. 2006. doi:10.1016/S0024-3205(01)01146-8.

MROZ, Z. et al. The effects of calcium benzoate in diets with or without organic acids on dietary buffering capacity, apparent digestibility, retention of nutrients, and manure characteristics in swine. Journal of Animal Science, v.78, p.2622-2632, 2000. Disponível em: <http://jas.fass.org/cgi/reprint/78/10/ 2622 ? $\mathrm{m} \mathrm{axt} \mathrm{o} \mathrm{s} \mathrm{h} \mathrm{o} \mathrm{w}=\&$ H I T S $=10$ \& h i t s $=1$ 0 \& R E S U L T F O R M A T $=\&$ s e a r c h i d $=1$ \&FIRSTINDEX=0\&volume=78\&firstpage=2622\&resourcetype=HWCIT>. Acesso em: 24 out. 2006.

NATIONAL RESEARCH COUNCIL. Nutrient requirements of poultry. 9.ed. Rev. ampl. Washington: National Academy, 1994. 93p. 
PARR INSTRUMENTS COMPANY. Instructions for the Parr 1720 Calorimeter Controller. Moline- IL, 1988. 35p. (Manual 165).

RAFACZ-LIVINGSTON, K.A. et al. The effects of various organic acids on phytate phosphorus utilization in chicks. Poultry Science, v.84, p.1356-1362, 2005. Disponível em: $<\mathrm{ht} \mathrm{tp}$ ://ps.fas s.org/cgi/reprint/84/9/ 1356 ? $\mathrm{maxt}$ oshow $=\&$ HITS $=10 \&$ hits $=10 \&$ RESULTF ORMAT $=\&$ andorexactfulltext $=$ and $\&$ searchid $=1 \&$ FIRS I N D E X $=0 \&$ s or t s p e c $=$ re levan c e $\&$ volu me $=8$ 4\&firstpage $=1356 \&$ resourcetype $=$ HWCIT $>$. Acesso em: 3 set. 2006 .

RAFACZ-LIVINGSTON, K.A. et al. Citric acid improves phytase phosphorus utilization in crossbred and commercial broiler chicks. Poultry Science, v.84, p.1370-1375, 2005. Disponível em: <http://ps.fass.org/cgi/reprint/84/9/ 1370 ? maxtoshow $=\&$ H I S $=10 \&$ hit s $=10 \&$ RESULTFORMAT $=\&$ and orexactfulltext $=$ an d \& searchid=1\&FIRSTINDEX=0\&sortspec=rele vance $\&$ volume $=84 \&$ firstpage $=1370 \&$ resourcetype $=$ HWCIT $>$. Acesso em: 10 nov. 2006.

RASCHKA, L.; DANIEL, H. Mechanisms underlying the effects of inulin-type fructans on calcium absorption in the large intestine of rats. Bone, v.37, p.728-735, 2005. Disponível em: <http://www.sciencedirect.com/ science?_ob=ArticleURL\&_udi=B6T4Y-4H0B T25 $1 \&$ _user $=687304 \&$ _coverDate $=11 \% 2 \mathrm{~F} 30 \% 2 \mathrm{~F} 2005 \&$ _alid $=101$

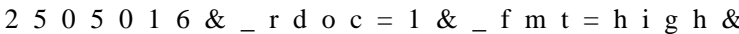

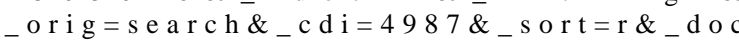
anchor $=\&$ view $=c \&$ ct $=1 \&$ _acct $=C 000037798 \&$ \& versio $\mathrm{n}=1 \&$ _ url versio n $=0 \&$ _ us e rid $=687304$ $\& m d 5=5 a 34 b 71$ ace5ee28ec209f6318cc60a10>. Acesso em: 30 jun. 2006. doi:10.1016/j.bone.2005.05.015.

ROSTAGNO, H.S. et al. Tabelas brasileiras para aves e suínos: composição de alimentos e exigências nutricionais. 2.ed. Viçosa: UFV, Departamento de Zootecnia, 2005. 186p.

SÁ, L.M. et al. Exigências nutricionais de cálcio para frangos de corte, na fase de crescimento e terminação. Revista Brasileira de Zootecnia, v.33, n.2, p.307-406, 2004. Disponível em: $<$ http://www.scielo.br/scielo.php?script=sci_arttext\&pid=S1516$35982004000200017 \& \operatorname{lng}=$ en\&nrm=iso $>$. Acesso em: 3 set. 2006. doi: 10.1590/S1516-35982004000200017.

SCHOULTEN, N.A. et al. Efeito dos níveis de cálcio da ração suplementada com fitase sobre a deposição de minerais na tíbia de frangos de corte de 22 a 42 dias. Revista Ciência e Agrotecnologia, v.27, n.1, p.206-210, 2003. Disponível em: $<$ http://www.editora.ufla.br/revista/27_1/art26.PDF>. Acesso em: 10 nov. 2006.

SEBASTIAN, S. et al. Efficiency of supplemental microbial phytase at different dietary calcium levels on growth performance and mineral utilization of broiler chickens. Poultry Science, v.75, p.1516-1523, 1996.

SNOW, J.L. et al. Phytase, citric acid, and 1-áhidroxycholecalciferol improve phytate phosphorus utilization in chicks fed a corn-soybean meal diet. Poultry Science, v.83, p.1187-1192, 2004. Disponível em: <http://ps.fass.org/cgi/reprint/ $83 / 7 / 1187$ ? maxtoshow $=\&$ HITS $=10 \&$ hits $=10 \&$ RESULTF ORMAT $=\&$ andorexactfulltext $=$ and $\&$ searchid $=1 \&$ F I R S T I N D E X $=0 \&$ s o r t s p e c $=$ rele v a n c e \& volume $=83 \&$ firstpage $=1187$ \& resourcetype $=$ HWCIT $>$. Acesso em: 24 jan. 2006.

TEDESCO, M.J. et al. Análises de solos, plantas e outros materiais. 2.ed. Rev. ampl. Porto Alegre: Departamento de Solos, UFRGS, 1995. 174p.

VALE, M.M. et al. Mixture of formic and propionic acid as additives in broiler feeds. Scientia Agrícola, v.61, n.4, p.371375, 2004. Disponível em: <http://www.scielo.br/ scielo.php? script =sci_arttext \& pid=S 0103 90162004000400004\&lng=en\&nrm=iso >. Acesso em:10 nov. 2006. doi: 10.1590/S0103-90162004000400004.

VALENCIA, Z.; CHAVEZ, E.R. Phytase and acetic acid supplementation in the diet of early weaned piglets: effect on performance and apparent nutrient digestibility. Nutrition Research, v.22, p.623-632, 2002. Disponível em: <http:// www.sciencedirect.com/science?_ob=ArticleURL\&_udi=B6TB145SR5B2-8\&_user $=687304 \&$ _coverDate $=05 \% 2$ F $31 \% 2$ F $2002 \&$ \& a lid = 1012534901 \&_rd o c $=1$ \& _ $\mathrm{fmt}=\mathrm{high} \&$ _orig $=$ search \&_cdi $=5129 \&$ _sort $=\mathrm{r} \&$ _ d o c a n c hor $=\&$ vi e w $=c_{-} \&$ c t $=1 \& \&_{\text {_ a c c t }=C}$ $000037798 \&$ _ version $=1 \&$ _ url Version $=0 \&$ userid $=687304 \& \mathrm{md} 5=2 \mathrm{~d} 2542 \mathrm{a} 096 \mathrm{af} 609 \mathrm{ebc} 1 \mathrm{~d} 279474 \mathrm{~d} 937 \mathrm{a} 6>$. Acesso em: 10 nov.2006. doi:10.1016/S0271-5317(02)00371-8.

VIEIRA, M.M. et al. Ácidos graxos de cadeia curta e fitase em dietas de frangos de corte com diferentes níveis de cálcio. In:REUNIÃO ANUAL DA SOCIEDADE BRASILEIRA DE ZOOTECNIA, 45., 2008. Lavras, MG. Anais... Lavras: Sociedade Brasileira de Zootecnia, 2008. CD-ROM. 